\title{
Combination a Skeleton Filter and Reduction Dimension of KernelPCA-Based on Palmprint Recognition
}

\author{
Muhammad Kusban ${ }^{1}$, Adhi Susanto ${ }^{2}$, and Oyas Wahyunggoro ${ }^{3}$ \\ ${ }^{1,2,3}$ Department of Electrical Engineering and Information Technology, Universitas Gadjah Mada, Indonesia \\ ${ }^{1}$ Department of Electrical Engineering, Univeristas Muhammadiyah Surakarta, Indonesia
}

\begin{tabular}{l}
\hline Article Info \\
\hline Article history: \\
Received Jun 29, 2016 \\
Revised Oct 18, 2016 \\
Accepted Nov 4, 2016 \\
\hline Keyword: \\
Skeleton \\
Kernel PCA \\
Palmprint recognition \\
Feature information \\
EER
\end{tabular}

Article Info

Article history:

Received Jun 29, 2016

Revised Oct 18, 2016

\begin{abstract}
Palmprint identification is part of biometric recognition, which attracted many researchers, especially when fusion with face identification that will be applied in the airport to hasten knowing individual identity. To accelerate the process of verification feature palms, dimension reduction method is the dominant technique to extract the feature information of palms. The mechanism will boost if the ROI images are processed prior to get normalize image enhancement. In this paper with three sample input database, a kernel PCA method used as a dimension reduction compared with three others and a skeleton filter used as a image enhancement method compared with six others. The final results show that the proposed method successfully achieve the target in terms of the processing time of 0.7415 second, the EER performance rate of $0.19 \%$ and the success of verification process about $99,82 \%$.
\end{abstract}

Copyright (c) 2016 Institute of Advanced Engineering and Science. All rights reserved.

\section{Corresponding Author:}

Muhammad Kusban

Departemen Teknik Elektro dan Teknologi Informasi (DTETI) - UGM

\& Teknik Elektro Universitas Muhammadiyah Surakarta

muhammadkusban.s3te13@mail.ugm.ac.id

Muhammad.Kusban@ums.ac.id

\section{INTRODUCTION}

Palmprint recognition is part of the biometric system which attracted many researchers. One way to optimize the verification and identification is to improve the appearance image of palm ROI (region of interest). The trick is to use a proper filter method to gain a uniform brightness level of the image so that the next process to obtain feature information becomes easier. Another trick is by selecting a dimension reduction method in accordance with the pattern of information from the palm so that the ongoing process can distinguish between the original pattern and the fake is more accurate and efficient in the terms of EER (equal error rate) and time.

There are some researchers who have discussed the use of the ROI filter and dimension reduction methods to improve the palmprint recognition. The filter function, among others, is to get the sharpness of the image [1] and also to access feature information more effectively [2]. Some researchers have used the filter method in the field are the Laplacian, the Gaussian, and the unsharp masking [3]. The method has a drawback that is by increasing the noise when an acquisition process takes place. By Wang and Leedham to eliminate the noise is by using a median filter that continued to use a 2D Gaussian law pass [4]. Zhao et al. using a series of filters, namely: match filter, the Wiener, and smoothing filter that the overall aims to elevate signal-to-noise ratio, remove noise, and to improve the appearance of the image [5].

Some researchers have used a dimension reduction algorithm to obtain an optimal palmprint recognition. In practice, the feature extraction of image taken directly from a 2D image matrix based instead of the vectors-based on scatter difference criterion. As a result, let to the small sample size (SSS) which will affect the appearance of the singularity problem. Wan has conducted research using two-dimensional graph embedding local discrimant analysis (2DLGEDA) to overcome the singularity LDA [6]. However, Xinchun found that the use of principal component analysis (PCA) is the best selection of dimension reduction compared with other 
algorithms [7]. The idea was reinforced by Imtiaz that for a more robust palmprint recognition, the use of PCA done after the process of 2D DWT in advance with the aim to get more efficient the local variables in each segment of palm [8].

From all of the dimension reduction methods which have been used in the study, none of them has an optimal performance on all side [9][10]. Therefore, in this paper offered a proposal to improve the detection system through palmprint with Kernel PCA (KPCA) that processed after the process skeleton filter in all ROI of palms. The KPCA is able to produce an efficient discriminant rate [11]. Although the preliminary research, Kusban [12] stated that Gabor parameters of $8 \times 5$ and the dimension reduction of PCA can gain a great achievement in verifying of palms.

From the research that have been conducted, the skeleton filter method for enhancement image and the KPCA for reduction dimension, produces a promising outcome. As a comparison, the skeleton filter compared with six other filters and the KPCA compared with three other dimension reduction. All simulations run using three kinds input of the database.

\section{PROPOSED METHOD}

\subsection{Image Enhancement}

Under normal condition, the entire acquisition image of palms can not be directly benefited to extract feature of palm ROI because it contains big unwanted information [2]. To solve the problem, image enhancement frequently is used to gain local pattern more clearly, so it helps in strengthening the output rate of feature information [13]. Some examples of the use of various filters to the ROI image of palms visible in Figure 1.

Filter skeleton has been previously used by Lin in his research to obtain all lines minutiae in palm [14]. The result is an ability of skeleton filter to improve the appearance of images, thus improving system performance and upgrade the point pattern matching approach. However, there is the weakness of this method that is increasing the number of feature information meaningless that resulted adds to the complexity of the computing process. To overcome this problem, the authors propose skeleton filter that only analyze the principal lines of palms by the threshold method which generate outcome as shown in Figure 1 (e).

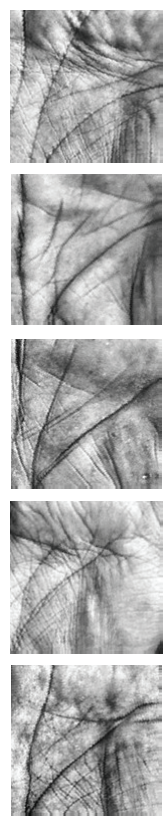

(a)
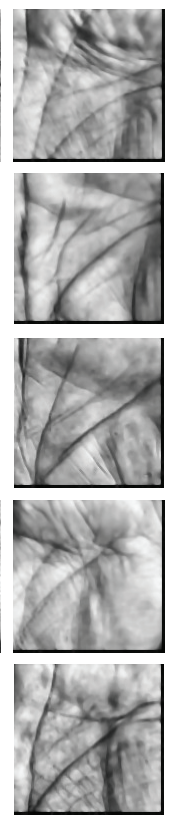

(b)
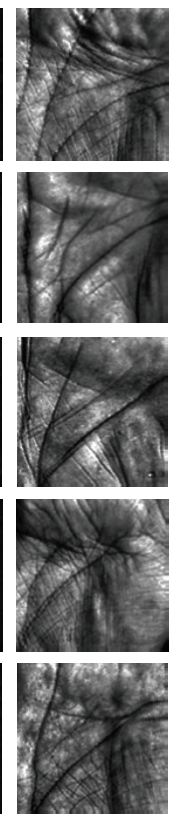

(c)
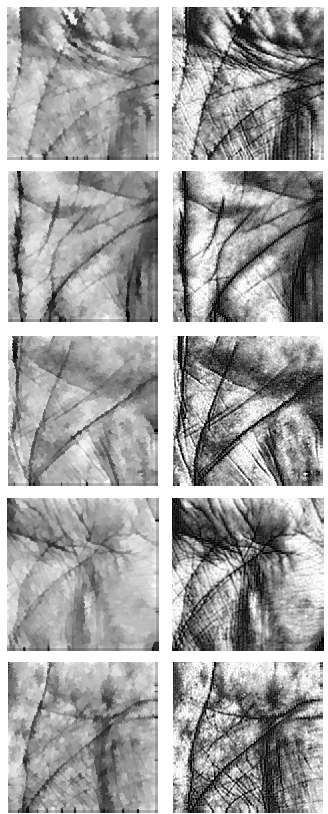

(d)
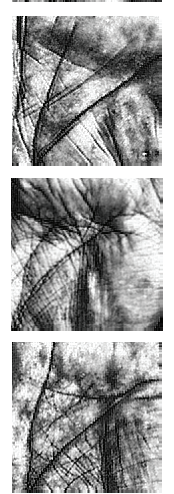

(e)
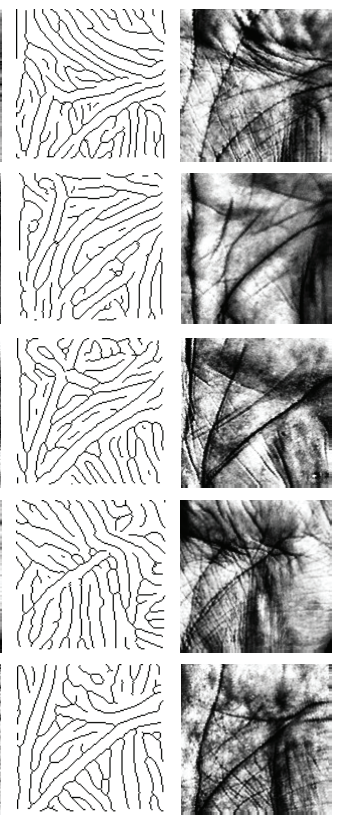

(f)

Figure 1: A series visualization ROI of palm from seven different filter process: (a) Original (b) Anisotropic (c) Multiple (d) Shock (e) Wavelet (f) Skeleton (d) Histogram Equalization.

The notation of the skeleton $S(\mathcal{G})$ from the set $\mathcal{G}$ is a point $p$ of $S(\mathcal{G})$ and $d_{p}$ is the biggest disk with center in $p$, then the $\mathcal{G}$ in this disc is a 'maximum disk'. The disk $d_{p}$ touches the boundary $\mathcal{G}$ in two or more 
different places. The skeleton of $\mathcal{G}$ is defined for morphology operation by the erosion and opening function.

$$
S(\mathcal{G})=\bigcup_{k=0}^{K} S_{k}(\mathcal{G})
$$

with $S_{k}(\mathcal{G})=(\mathcal{G} \ominus k \mathcal{H})-(\mathcal{G} \ominus k \mathcal{H}) \circ \mathcal{H}$ and $\mathcal{H}$ is the matrix dilation and erotion or structuring element with $(\mathcal{G} \ominus k \mathcal{H})$ for $k$ sequential erosions of $\mathcal{G}:(\mathcal{G} \ominus k \mathcal{H})=(\ldots((\mathcal{G} \ominus \mathcal{H}) \ominus \mathcal{H}) \ominus \ldots) \ominus \mathcal{H}, k$ times, and $K$ is the last iterative process before $\mathcal{G}$ erode to an empty value, or $K=\max \{k \mid(\mathcal{G} \ominus k \mathcal{H}) \neq \emptyset\}$.

In its application, the filter skeleton is approached by using the principles distance between each point and their boundary. If known the initial $x_{0}\left(m_{1}, n_{1}\right)=x\left(m_{2}, n_{2}\right)$ are two points equidistant, then the distance transform defined

$$
x_{k}\left(m_{2}, n_{2}\right)=x_{0}\left(m_{1}, n_{1}\right)+\min \left\{x_{k-1}(i, j): d\left(m_{1}, n_{1} ; i, j\right) \leq 1\right\}
$$

The skeleton is the set of value whose distance from the nearest boundary is has maximum in locally.

$$
\left\{\left(m_{1}, n_{1}\right): x_{k}\left(m_{2}, n_{2}\right) \geq x_{k}(i, j), d\left(m_{1}, n_{1}, i, j\right) \leq 1\right\}
$$

\subsection{Kernel PCA}

The main concern in biometric recognition is the amount of data continued increase significantly. It is why the dimension reduction method is absolutely necessary. In an effort to divide the data into smaller, the PCA method is widely used in palmprint recognition [15]. However, the technique has drawbacks difficult to acknowledge the feature information in a single image that have a variation orientation. To overcome this problem, it is necessary to use non-linear method and kernel PCA (KPCA) successfully used in biometrics [11]. If it is known in the PCA apply an association law $C_{v} w=\lambda w$, with $C_{v}$ is the matrix covariance and $x$ is the center data, then:

$$
C_{v}=\frac{1}{n} \sum_{i=1}^{q} x_{i} x_{i}^{\prime}
$$

under the condition $w \in\left\{x_{1}, \ldots, x_{n}\right\}$ if $\lambda \neq 0, \lambda<x_{i}, w \geq x_{i}$, and $C_{v} w>i=1, \ldots, q$. When the $\Phi$ is a high-dimension space, then the KPCA applies the same situations. $C_{v}=\frac{1}{q} \sum_{i=1}^{q} \Phi\left(x_{i}\right) \Phi\left(x_{i}\right)^{T}$. For kernel matrix $K$ with size $q \times q$ will have a value of association $k\left(x_{i}, x_{j}\right)=\left\langle\Phi\left(x_{i}\right), \Phi\left(x_{j}\right)\right\rangle$, with the centering data is as follow

$$
\hat{\Phi}(x)=\Phi(x)-\Phi_{s}(x)=\Phi(x)-\frac{1}{q} \sum_{i=1}^{q} \Phi\left(x_{i}\right),
$$

thus, the transformation kernel space is

$$
\begin{aligned}
\hat{k}(x, z) & =\langle\hat{\Phi}, \hat{\Phi}(z)\rangle \\
& =\left\langle\Phi(x)-\frac{1}{q} \sum_{i=1}^{q}, \hat{\Phi}(z)-\frac{1}{q} \sum_{i=1}^{q} \Phi\left(x_{i}\right)\right\rangle \\
& =k(x, z)-\frac{1}{q} \sum_{i=1}^{q} k\left(x, x_{1}\right)-\frac{1}{q} \sum_{i=1}^{q} k\left(z, x_{i}\right)+\frac{1}{q^{2}} \sum_{i, j=1}^{q} k\left(x_{i}, x_{j}\right)
\end{aligned}
$$

\section{RESEARCH AND DISCUSSION}

Research conducted using three database input of palm image, namely: Casia $(\mathcal{C})$, IITD India $(\mathcal{I})$, and PolyU $(\mathcal{P})$. The number of data samples, respectively, are 550, 450, and 650 with each item have a variety of image appearance as much as 5, 6, and 10. So that the total amount of imagery used is 11.950 palm images that different from each other. Software for simulation is Matlab R2014b under Windows 7 Pro. While, the hardware is PC Intel i $4500 \mathrm{~K}$ with $8 \mathrm{GHz}$ of main memory. 
From the research that has been done for palmprint recognition, a rate of research shown in Table 1. Four types reduction dimension, namely KPCA, KFA, LDA, and PCA are used to get feature information of palm image from three different databases and seven distinct filter. The results process from seventh filter is shown in Fig 1. Finally, The result of research are the rate process and the performance of EER value from each filters.

Table 1: The results of using multiple filters in four dimension reduction based (RD) to obtain a rate of time process and rate of performance $(\mathrm{EER})$ from three types databases: Casia $(\mathcal{C})$, IITD India $(\mathcal{D})$, and PolyU $(\mathcal{P})$

\begin{tabular}{|c|c|c|c|c|c|c|c|c|c|c|}
\hline \multirow{2}{*}{$\mathcal{D R}$} & \multirow{2}{*}{ Method } & \multicolumn{3}{|c|}{ Casia } & \multicolumn{3}{|c|}{ IITD-India } & \multicolumn{3}{|c|}{ PolyU } \\
\hline & & Time & EER & Ver. & Time & EER & Ver. & Time & EER & Ver. \\
\hline \multirow[t]{7}{*}{ KFA } & Original & 1,8587 & 0,4261 & 0,5746 & 0,5868 & 0,3363 & 0,6656 & 1,1003 & 0,3011 & 0,6991 \\
\hline & Anisotropic & 1,7318 & 0,4807 & 0,5215 & 0,5742 & 0,3364 & 0,6644 & 1,0355 & 0,3771 & 0,6227 \\
\hline & Multiple & 1,8023 & 0,4175 & 0,5823 & 0,5626 & 0,3376 & 0,6622 & 1,1562 & 0,3307 & 0,6700 \\
\hline & Shock & 1,7188 & 0,4696 & 0,5315 & 0,5709 & 0,3998 & 0,6000 & 1,1946 & 0,3984 & 0,6009 \\
\hline & Wavelet & 1,7338 & 0,4709 & 0,5292 & 0,5935 & 0,4278 & 0,5722 & 1,1024 & 0,4145 & 0,5855 \\
\hline & Skeleton & 1,7303 & 0,4446 & 0,5554 & 0,5666 & 0,3174 & 0,6822 & 1,1060 & 0,3502 & 0,6500 \\
\hline & Histogram & 1,7513 & 0,4472 & 0,5531 & 0,6010 & 0,3067 & 0,6922 & 1,1536 & 0,2787 & 0,7209 \\
\hline \multirow[t]{7}{*}{ KPCA } & Original & 1,3050 & 0,0249 & 0,9754 & 0,4066 & 0,0189 & 0,9811 & 0,7514 & 0,0036 & 0,9964 \\
\hline & Anisotropic & 1,3502 & 0,2267 & 0,7739 & 0,4225 & 0,5000 & 0,4611 & 0,7368 & 0,5000 & 0,5755 \\
\hline & Multiple & 1,3572 & 0,5000 & 0,5085 & 0,4191 & 0,0200 & 0,9800 & 0,7676 & 0,0027 & 0,9973 \\
\hline & Shock & 1,2729 & 0,0109 & 0,9892 & 0,3978 & 0,0233 & 0,9767 & 0,7873 & 0,0073 & 0,9927 \\
\hline & Wavelet & 1,2697 & 0,0663 & 0,9339 & 0,4163 & 0,0434 & 0,9567 & 0,7717 & 0,0084 & 0,9918 \\
\hline & Skeleton & 1,3039 & 0,0146 & 0,9854 & 0,4073 & 0,5000 & 0,4933 & 0,7415 & 0,0019 & 0,9982 \\
\hline & Histogram & 1,3331 & 0,0154 & 0,9846 & 0,4053 & 0,0178 & 0,9822 & 0,9600 & 0,0025 & 0,9973 \\
\hline \multirow[t]{7}{*}{ LDA } & Original & 4,1741 & 0,0123 & 0,9877 & 2,0252 & 0,0148 & 0,9856 & 3,1772 & 0,0037 & 0,9964 \\
\hline & Anisotropic & 4,2425 & 0,2405 & 0,7592 & 2,1251 & 0,0199 & 0,9800 & 3,1990 & 0,0082 & 0,9918 \\
\hline & Multiple & 4,3174 & 0,0169 & 0,9831 & 2,0290 & 0,0166 & 0,9833 & 3,3382 & 0,0046 & 0,9955 \\
\hline & Shock & 4,2774 & 0,0129 & 0,9869 & 2,0957 & 0,0266 & 0,9733 & 3,2568 & 0,0073 & 0,9927 \\
\hline & & 4,2314 & 0,0646 & 0,9354 & 2,1914 & 0,0557 & 0,9444 & 3,4504 & 0,0136 & 0,9864 \\
\hline & Skeleton & 4,2408 & 0,0093 & 0,9908 & 2,0870 & 0,0167 & 0,9833 & 3,3474 & 0,0036 & 0,9964 \\
\hline & Histogram & 4,3522 & 0,0094 & 0,9908 & 2,0972 & 0,0167 & 0,9833 & 3,3670 & 0,0025 & 0,9973 \\
\hline \multirow[t]{7}{*}{ PCA } & Original & 3,1740 & 0,0347 & 0,9654 & 1,6684 & 0,0224 & 0,9778 & 2,0115 & 0,0079 & 0,9918 \\
\hline & Anisotropic & 3,2567 & 0,2732 & 0,7269 & 1,4563 & 0,0256 & 0,9744 & 2,1097 & 0,0112 & 0,9891 \\
\hline & Multiple & 3,3113 & 0,0308 & 0,9692 & 1,4778 & 0,0244 & 0,9756 & 2,1430 & 0,0064 & 0,9936 \\
\hline & Shock & 3,3131 & 0,0324 & 0,9677 & 1,4440 & 0,0256 & 0,9744 & 2,2786 & 0,0091 & 0,9909 \\
\hline & Wavelet & 3,1949 & 0,1033 & 0,8969 & 1,4141 & 0,0656 & 0,9344 & 2,0819 & 0,0199 & 0,9800 \\
\hline & Skeleton & 3,2092 & 0,0215 & 0,9785 & 1,4256 & 0,0201 & 0,9800 & 2,2206 & 0,0046 & 0,9955 \\
\hline & Histogram & 3,2495 & 0,0223 & 0,9777 & 1,4204 & 0,0210 & 0,9789 & 2,1732 & 0,0046 & 0,9955 \\
\hline
\end{tabular}

The values listed in the Table 1 directly show that the skeleton filter is superior at the time consumed compared others on three different databases. It is seen that the use of skeleton contains the same kind balanced line thickness with the color of white in the background. While the other type has lines different thickness with dark, distinct gradation of the background. In Figure 2 show some of the performance curve ROC (the receiver operating characteristic), CMC (the cumulative match curve), DET (the detection error tradeoff), and EPC (the expected performance curve). Seen from the display of four curves, the skeleton filter with a yellow color representation dominate other types. Although the ROC curve and the DET in Figure 2 (a) and (c) the skeleton inferior to shock filter, but eventually the method has best value and the statement is reinforced with a view EPC curve shown in Figure 2 (d) that the yellow color is the bottom line or has an error rate of the smallest.

The next process is the selection method of dimension reduction after all ROI image of palms filtered by the skeleton method. A KPCA (the kernel principal component analysis) method has the outstanding performance compared with other reduction dimension such as: LDA (linear discriminant analysis), KFA (kernel fisher analysis) and PCA (principal component analysis) as seen in the Figure 3. The KPCA method with black color lines appear to have the most excellent display of curve performance ROC, CMC, DET, and EPC. Especially for ROC in Figure 3 (a), where the black color lines is in the top position curve so that the false rejection 
Table 2: Performance of proposed system and other systems

\begin{tabular}{clr}
\hline No. & Type & EER (\%) \\
\hline 1. & Various noise density levels of palms on phase-difference information, [16] & 5.8475 \\
2. & Wavelet combination on new wavelet based method, [17] & 4.0702 \\
3. & Fused on SIFT-based Image Alignment, [18] & $0.4846 \& 0.5078$ (left-right) \\
4. & PV-Full-1.0 by FVC-onGoing on Latent Palmprint Matching, [19] & 5.6 \\
5. & EER on Multifeature-Based High-Resolution, [20] & 4.8 \\
6. & Proposed approach & 0.19 \\
\hline
\end{tabular}

rate has the highest value or close to 1. While the EPC curve in Figure 3 (d)looks the black color lines to be in the lowest position error rate or highest percentage of verification. The statement reinforced by evidence from Table 1 that the use of KPCA method even robust since data input increasing in number ranging from small to larger that is IITD India, Casia, and then PolyU. From the output research, it yields better performance in terms of error equal rate (EER) when compared with other similar studies as shown in Table 2.

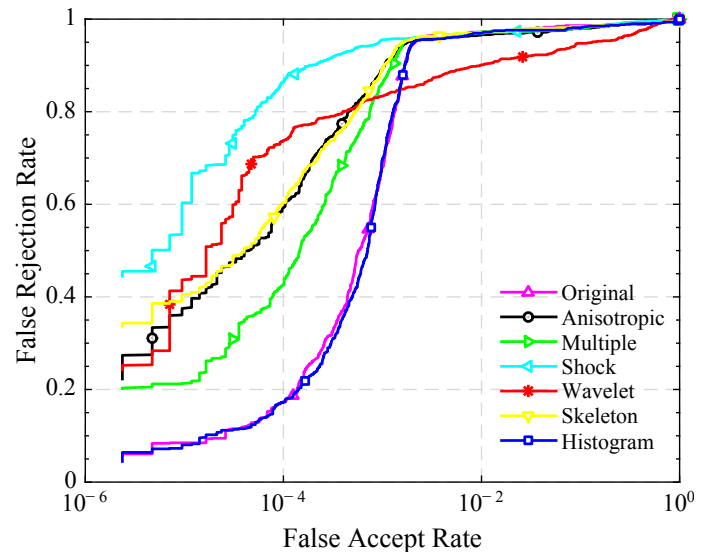

(a)

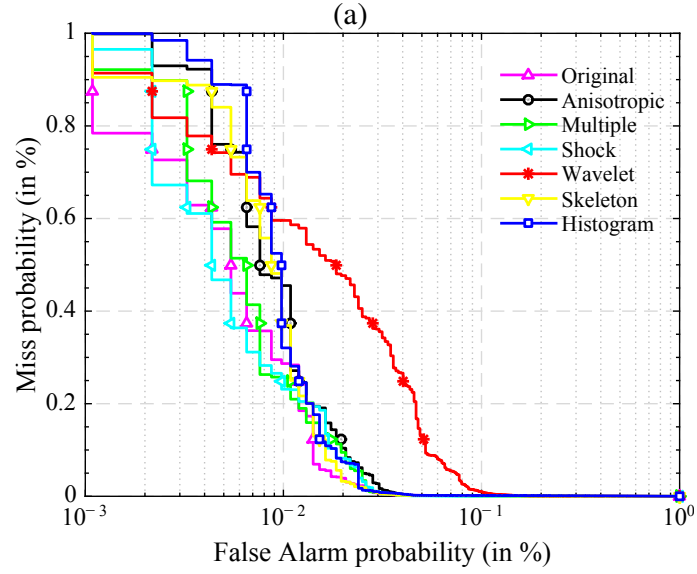

(c)

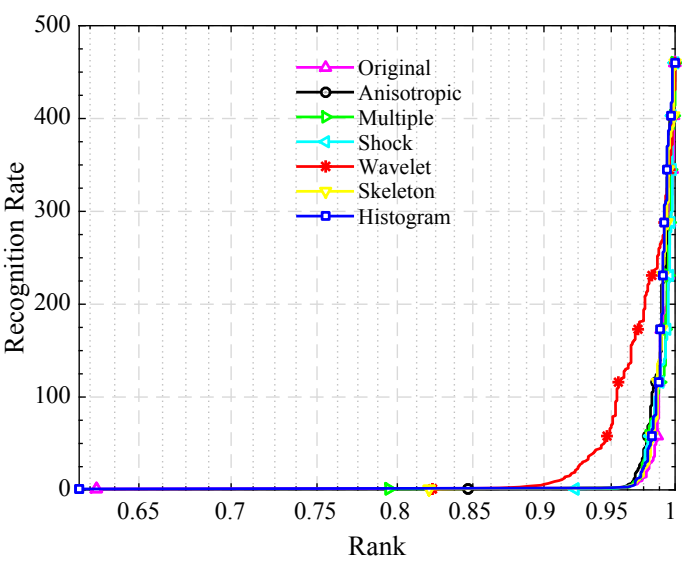

(b)

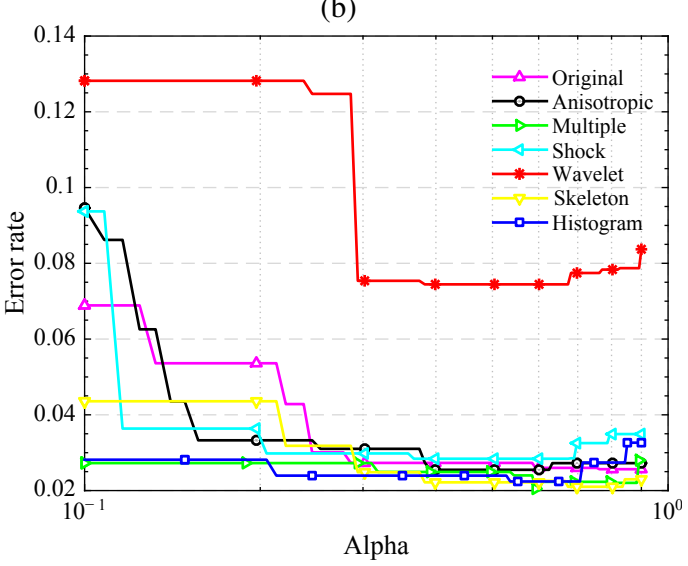

(d)

Figure 2: Curves are used to show the achievement of various the filter method in ROI image including the type of performance to: (a) ROC (b) CMC (c) DET (d) EPC.

\section{CONCLUSION}

Globally, the skeleton method has the best performance compared to other filters: original, anisotropic, multiple, shock, wavelet, and histogram with the highest value is $99,82 \%$ in successfully verification process with error rate about $0,19 \%$ in PolyU database and KPCA-based. Overall the KPCA is the most suitable method of dimension reduction to obtain the feature from verification and identification of palms. 

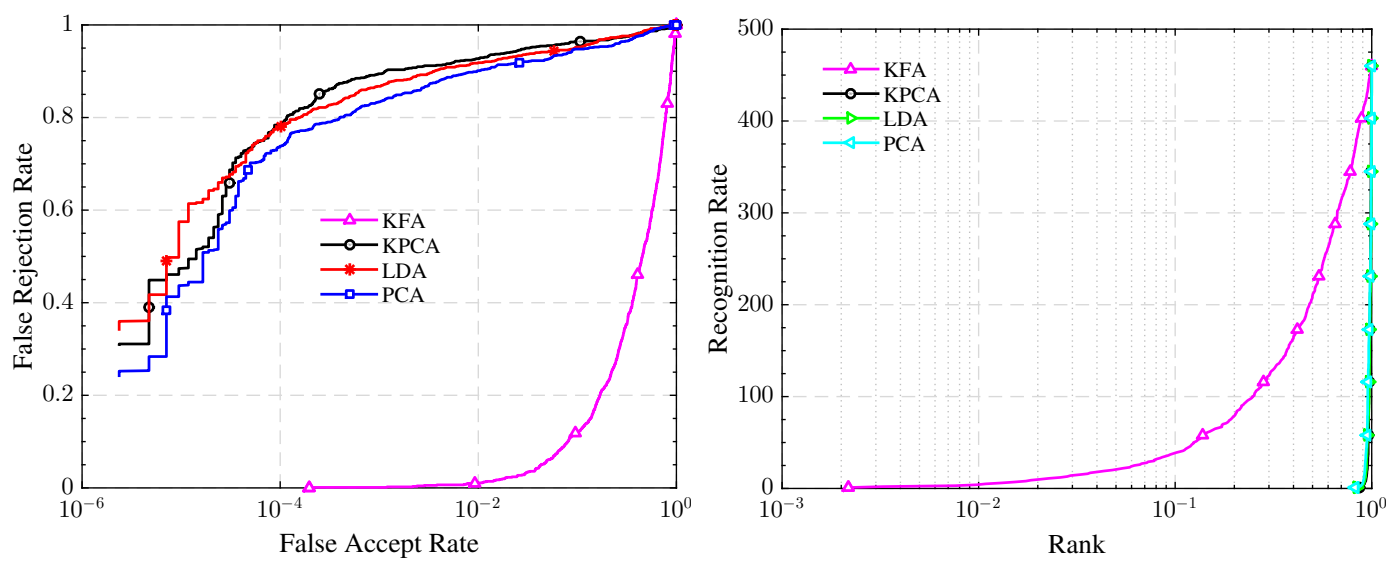

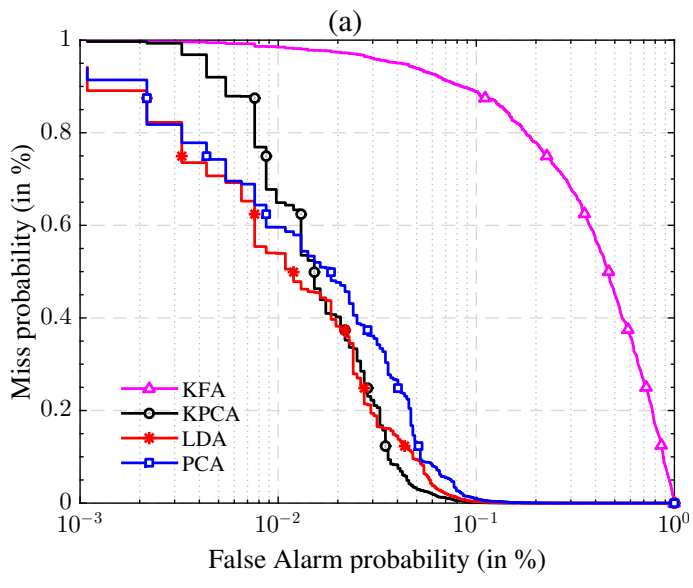

(c)

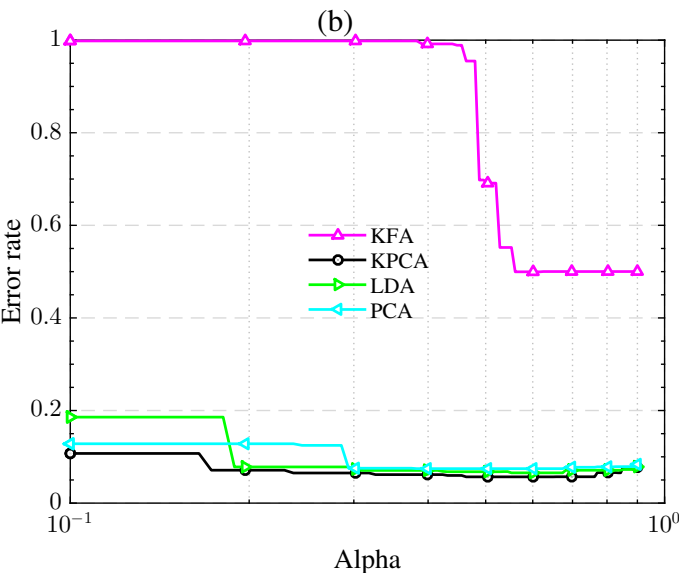

(d)

Figure 3: Curves are used to show the achievement of various the dimension reduction method in palmprint recognition including the type of performance to: (a) ROC (b) CMC (c) DET (d) EPC.

\section{REFERENCES}

[1] Goh Kah Ong Michael, Tee Connie, and Andrew Beng Jin Teoh. A contactless biometric system using multiple hand features. J. Vis. Comun. Image Represent, 23:1068-1084, 2012.

[2] C. Lakshmi Deepika et al. Palmprint authentication using modified legendre moments. Procedia Computer Science, pages 164-172, 2010.

[3] O. Nibouche and J. Jiang. Palmprint matching using feature points and svd factorisation. Digital Signal Processing: A Review Journal, 23:1154-1162, 2013.

[4] L. Wang et al. Infrared imaging of hand vein patterns for biometric purposes. IET Computer Vision, 1:113-122, 2007.

[5] S. Zhao et al. Extracting hand vein patterns from low-quality images: A new biometric technique using low-cost devices. Image and Graphics, 2007. ICIG 2007. Fourth International Conference on, 2007.

[6] Wan et al. Fuzzy two-dimensional local graph embedding discriminant analysis (f2dlgeda) with its application to face and palm biometrics. Neural Computing and Applications, 23:201-207, 2013.

[7] Xinchun et al. Palmprint recognition based on curvelet transform decision fusion. Procedia Engineering, 23:303-309, 2011.

[8] Imtiaz et al. A wavelet-based dominant feature extraction algorithm for palm-print recognition. Digital Signal Processing: A Review Journal, 23:244-258, 2013.

[9] Yong Xu et al. A feature extraction method for use with bimodal biometrics. Pattern Recognition, 43:1106-1115, 2010.

[10] Milad Jafari Barani. et al., "implementation of gabor filters combined with binary features for gender recognition". International Journal of Electronical and Computer Engineering (IJECE), 4:108-115, 2013.

[11] M. Ekinci and M. Aykut. Gabor-based kernel pca for palmprint recognition. Electronics Letters, 43:1077-1079, 2007.

[12] M. Kusban. Verifikasi dan identifikasi telapak tangan dengan kernel gabor. Jurnal Nasional Teknik Elektro dan Teknologi Informasi (JNTETI), 4:2, Mei 2015.

[13] Wang et al. Discriminative and generative vocabulary tree: With application to vein image authentication and recognition. Image and Vision Computing, 34:51-62, 2015. 
[14] J. Li and G. Shi. A novel palmprint feature processing method based on skeleton image. Signal Image Technology and Internet Based Systems, 2008. SITIS '08. IEEE International Conference on, pages 221-228, 2008.

[15] Sara Zokaee and Karim Faez. Human identification based on electrocardiogram and palmprint. International Journal of Electronical and Computer Engineering (IJECE), 2:261-266, 2012.

[16] G. S. Badrinath and Phalguni Gupta. Palmprint based recognition system using phase-difference information. Future Generation Computer Systems, 28(1):287-305, 2012.

[17] Naresh Bhuma and C H Himabin. N EW PALM PRINT AUTHENTICATION SYSTEM BY USING WAVELET BASED METHOD. 2(1), 2011.

[18] Wei Bu, Qiushi Zhao, and Xiangqian Wu. SIFT-based Image Alignment for Contactless Palmprint Verification. pages $1-6,2013$.

[19] J. Dai and J. Zhou. Multifeature-based high-resolution palmprint recognition. IEEE Transactions on Pattern Analysis and Machine Intelligence, 33(5):945-957, May 2011.

[20] E. Liu, A. K. Jain, and J. Tian. A coarse to fine minutiae-based latent palmprint matching. IEEE Transactions on Pattern Analysis and Machine Intelligence, 35(10):2307-2322, Oct 2013. 